\title{
Evaluación de la inmunogenicidad de una proteína recombinante de una Pasteurella multocida aislada de alpacas con neumonía
}

\author{
EVALUATION OF THE IMMUNOGENICITY OF A RECOMBINANT PROTEIN OF A Pasteurella \\ multocida ISOLATED FROM ALPACAS WITH PNEUMONIA
}

\author{
Jorge Maximiliano G. ${ }^{1}$, Lenin Maturrano H., ${ }^{1,2,4}$, Luis Luna E. ${ }^{1}$, \\ Raquel Hurtado C. ${ }^{1}$, Ana Chero O. ${ }^{1}$, Raúl Rosadio A. ${ }^{1,3}$
}

\section{Resumen}

El objetivo del estudio fue evaluar la actividad inmunogénica in vitro de una proteína P6-like recombinante procedente de un cultivo de Pasteurella multocida aislada de cuadros de neumonía en crías de alpacas. Se desafiaron cultivos de células mononucleares periféricas sanguíneas (PBMC) con una proteína recombinante P6-like a una concentración de 10 ng por muestra, desde las 3 hasta las 72 horas. Se extrajo ARN totales para realizar la prueba de RT-PCR en tiempo real para observar los niveles de expresión de citoquinas de la respuesta inmune Th1 (TNF- $\alpha$, IFN- $\gamma$ e IL-2) y Th2 (IL-10 e IL-4) en los tiempos establecidos. Se encontró que tanto las citoquinas con perfil Th1 como Th2 expresan un mayor número de veces con respecto a PBMC no expuestos a la proteína recombinante, siendo las 24 y 48 horas los momentos de mayor expresión. Asimismo, se encontró una aparente tendencia hacia el perfil Th2, pero en niveles que no influyen en la expresión de citoquinas del otro perfil.

Palabras clave: Pasteurella multocida; P6-like; alpaca; RT-PCR en tiempo real; cuantificación relativa

\footnotetext{
${ }^{1}$ Laboratorio de Microbiología y Parasitología Veterinaria, ${ }^{2}$ Laboratorio de Zootecnia y Producción Agropecuaria, Facultad de Medicina Veterinaria, Universidad Nacional Mayor de San Marcos, Lima, Perú

${ }^{3}$ CONOPA - Instituto de Investigación y Desarrollo de Camélidos Sudamericanos, Lima, Perú

${ }^{4}$ E-mail: amaturranoh@unmsm.edu.pe
}

Recibido: 19 de junio de 2017

Aceptado para publicación: 28 de noviembre de 2017 
The aim of the study was to evaluate the in vitro immunogenic activity of a recombinant P6-like protein from a culture of Pasteurella multocida isolated from pneumonia cases in young alpacas. Blood peripheral mononuclear cells (PBMCs) were challenged with a recombinant P6-like protein at a concentration of $10 \mathrm{ng}$ per sample, from 3 to 72 hours. Total RNA was extracted to perform the real-time RT-PCR test to observe cytokine expression levels of the Th1 immune response (TNF- $\alpha$, IFN- $\gamma$ and IL-2) and Th2 (IL-10 and IL-4) in the established times. Both Th1 and Th2 profile cytokines expressed a greater number of times than PBMC not exposed to the recombinant protein, where at 24 and 48 hours showed the greater expressions. Likewise, an apparent trend toward the Th2 profile was found, but at levels that did not influence the expression of cytokines in the other profile.

Key words: Pasteurella multocida; P6-like; alpaca; RT-PCR Real Time; relative quantification

\section{INTRODUCCIÓN}

La alpaca, una de las cuatro especies de camélidos sudamericanos, es sustento económico de numerosas familias en las regiones altoandinas del Perú (Ameghino y DeMartini, 1991). Los problemas de manejo en la crianza de la alpaca, especialmente las altas tasas de mortalidad neonatales que pueden superar el 50\% limitan el desarrollo de esta industria pecuaria y afectan la economía de los criadores (Ameghino y DeMartini, 1991; Bustinza, 2001). Las principales causas de mortalidad neonatal son las causas infecciosas, siendo las neumonías aquellas de mayor casuística (Garmendia et al., 1986; Ameghino y DeMartini, 1991; Bustinza, 2001; Cirilo et al., 2012).

Las neumonías son causadas por diversos agentes patógenos, entre virales y bacterianos, que coexisten en procesos infecciosos generando cuadros de neumonías agudas. Entre ellos, Pasteurella multocida es la principal bacteria involucrada en las infecciones neumónicas en alpacas, seguida de Mannhemia haemolytica que, individualmente o en conjunto con otros agentes infecciosos, generan cuadros hiperagudos en crías durante la época de parición, afectando también a crías más grandes en épocas de estrés como el destete y la esquila (Rosadio et al., 1990; Cirilo et al., 2012; Guzmán et al., 2013).

P. multocida se le clasifica en serogrupos (A, B, D, E y F) basados en sus antígenos capsulares; pero además es clasificada en serotipos de acuerdo con el lipopolisacárido presente, usando el test de Heddleston (Heddleston et al., 1972; Harper et al., 2006, 2015). P. multocida cuenta con una variedad de factores de virulencia que juegan roles importantes en la patogénesis de la enfermedad como, por ejemplo, en la evasión de la respuesta inmune y la captación de hierro, entre otros (Harper et al., 2006).

Las proteínas de membrana externa (OMP) son estructuras muy importantes en la patogénesis de la enfermedad. Muchos de estos compuestos están involucrados en la captación de hierro y en la adhesión, lo cual es importante para el establecimiento y supervivencia de la bacteria (Boyce et al., 2012). Varias OMP son inmunógenos y los anticuerpos producidos contra estas OMP demuestran una fuerte acción protectora. Tales antígenos se pueden usar como com- 
ponentes de vacunas de subunidades. La inmunogenicidad de ciertas OMP de $P$. multocida ha sido demostrada en terneros (Kedrak y Borkowska, 2003), conejos (Lu et al., 1991) y gallinas (Zhang et al., 1994). Otros estudios indican que una OMP similar a una proteína de adhesión P6 de Haemophilus influenzae (P6-like) induce a una mejor protección al inducir una respuesta significativa en producción de anticuerpos específicos, lo que lo convierte en un inmunógeno adecuado para ser usado en vacunas (Kasten et al., 1995; Shivachandra et al., 2017).

El presente estudio buscó determinar el perfil inmunogénico Th1 y Th2 de una proteína de membrana externa P6-like recombinante de $P$. multocida aislada de casos neumónicos fatales en alpacas obtenida mediante la metodología de la vacunología reversa, como parte de la elaboración de una vacuna de última generación para la prevención de neumonías pasteurelósicas en crías de alpacas.

\section{Materiales y MéTodos}

\section{Lugar del Estudio}

Los ensayos in vitro se realizaron en el Laboratorio de Biología y Genética Molecular de la Facultad de Medicina Veterinaria (FMV) de la Universidad Nacional Mayor de San Marcos (Lima, Perú).

\section{Proteína Recombinante P6-like}

En los ensayos experimentales se usó una proteína recombinante P6-like (proteína de membrana externa) producida en la FMV. La elección de esta proteína como candidato vacunal fue parte del desarrollo del Proyecto Innovate Perú (FINCyT) Contrato N. ${ }^{\circ}$ 133-FINCyT-IB-2013, el cual busca realizar una vacuna de nueva generación para la prevención y control de las neumonías pasteurelósicas en alpacas. Por ello, se aisló una Pasteurella multocida de casos neumónicos en crías de alpacas de la sierra sur del país (Puno) y se realizó la secuenciación de su genoma.

A partir de la secuenciación del genoma, se evaluó y comparó in silico con datos disponibles del genoma de $P$. multocida en el NCBI, VFDB (Virulence Factors DataBase), MvirDB (Microbial Virulence DataBase) y Protegen, además de usar diversos programas (Glimmer v. 3.02, CAP3, Transeq, BLAST v. 2.2.27, Psortb v. 3.02, SignalP v. 4.0, LipoPv. 1.0, TMHMM v. 2.0), identificándose 10 candidatos; entre ellos el gen que codifica la P6-like. Mediante el uso de cebadores específicos (Cuadro 1) fue amplificado mediante PCR a partir de aislados de $P$. multocida procedentes de cuadros neumónicos en alpacas. Posteriormente, mediante el uso del kit Champion ${ }^{T M} p E T$ Directional TOPO ${ }^{\circledR}$ Expression Kit with BL21 Star ${ }^{\mathrm{TM}}$ (DE3) One Shot ${ }^{\mathrm{TM}}$ Chemically Competent E. coli (Invitrogen) se clonó y expresó en cepas de E. coli BL21 ${ }^{\mathrm{TM}}$, especial para este tipo de técnicas. Por último, la proteína fue purificada mediante el Kit ProBond ${ }^{\mathrm{TM}}$ Puryfication System y conservada en refrigeración.

\section{Células Mononucleares Periféricas San- guíneas (PBMC)}

Para la extracción de las PBMC se usaron alpacas Huacaya hembras de 3 años, clínicamente sanas, del Laboratorio de Reproducción de la FMV. Los animales se encontraban separados de otras especies animales y eran alimentados con heno y agua ad libitum.

Se recolectaron $30 \mathrm{ml}$ de sangre periférica de un animal en tubos Vacutainer ${ }^{\circledR}$ con anticogulante (EDTA), mediante punción de la vena yugular. La sangre fue centrifugada en gradiente de densidad usando el reactivo Ficoll-Paque (Sigma), siguiendo las instrucciones del fabricante. Para los 
Cuadro 1. Secuencias, tamaño, temperaturas de annealing y referencias de las citoquinas usadas para la evaluación inmunogénica en alpacas

\begin{tabular}{|c|c|c|c|c|}
\hline & Genes & Secuencias & $\begin{array}{l}\text { Productos } \\
\text { amplificados } \\
\text { (bp) }\end{array}$ & $\mathrm{Tm}$ \\
\hline \multirow{6}{*}{ Th1 } & \multirow{2}{*}{ IFN- $\gamma$} & 5'-ATTGTCTCCTTCTACTTCAA-3' & \multirow{2}{*}{258} & \multirow{2}{*}{45} \\
\hline & & 5'-AGCGGAAGAGAAGTCAGAAT -3' & & \\
\hline & \multirow{2}{*}{$\mathrm{TNF} \alpha$} & 5'-CTACTCCCAGGTCCTCCTGA-3' & \multirow{2}{*}{251} & \multirow{2}{*}{60} \\
\hline & & 5'-GGTAGTTGGGCATGTTGATC-3' & & \\
\hline & \multirow{2}{*}{ IL-2 } & 5'-AAACTCTCCAGGATGCTCAC-3' & \multirow{2}{*}{202} & \multirow{2}{*}{49} \\
\hline & & 5'-GGAACTGAAGGGATCTGAAA-3' & & \\
\hline \multirow{4}{*}{ Th2 } & \multirow{2}{*}{ IL-4 } & 5'-CAAAGAACACAACTGAGAAG-3' & \multirow{2}{*}{203} & \multirow{2}{*}{46} \\
\hline & & 5'-GGCTAAAGAAGATTATGAAG-3' & & \\
\hline & \multirow{2}{*}{ IL-10 } & 5'-AAGCCTTGTCGGAGATGAC-3' & \multirow{2}{*}{246} & \multirow{2}{*}{55} \\
\hline & & 5'-AGCCATGAGTGAGTTCGACA-3' & & \\
\hline \multirow{2}{*}{ Normalizador } & \multirow{2}{*}{ GAPDH } & 5'-GTGAAGGTCGGAGTGAACG-3' & \multirow{2}{*}{356} & \multirow{2}{*}{60} \\
\hline & & 5'-GAGATGATGACCCTCTTGGC-3' & & \\
\hline
\end{tabular}

Las referencias para Th1 - IFN- $\gamma$, TNFa e IL-2 son Odbileg et al. (2008, 2005 y 2006), respectivamente, para Th2 es Odbileg et al. (2006) y para el normalizador es Patil et al. (2004)

lavados de las células mediante centrifugación se usó PBS $1 \mathrm{X}$ pH 7.8 y luego se suspendieron en medio de cultivo RPMI (Roswell Park Memorial Institute medium) 1640 con L-Glutamina (Sigma), adicionando $100 \mathrm{IU} / \mathrm{mL}$ de penicilina (Sigma) y $10 \% \mathrm{de}$ suero fetal bovino (SFB) (Sigma) inactivado por calor. Se uniformizó la muestra mediante diluciones con RPMI 1640 a una concentración de $1 \times 10^{6}$ células $/ \mathrm{ml}$. Uniformizado el cultivo, se colocó $1200 \mu \mathrm{l}$ de cultivo celular final por pocillo en una placa de poliestireno.

\section{Evaluación in vitro de la Proteína Recombinante P6-like}

Una vez preparado los cultivos celulares de PBMC se formaron dos grupos: calibrador y desafío. El grupo calibrador conformado por tres muestras $(1200 \mu 1$ por muestra), no fueron expuestas a estímulos y su perfil citocínico fue evaluado a las $0 \mathrm{~h}$ del experimento. El grupo desafío conformado por cultivos celulares $(1200 \mu 1$ por muestra, $1 \times 10^{6}$ células $/ \mathrm{ml}$ ) fue inoculado con $10 \mathrm{ng}$ de proteína recombinante $\mathrm{P} 6$-like, evaluándose a las $3,6,9,12,18,24,48$ y $72 \mathrm{~h}$ por triplicado. Las muestras se mantuvieron en una incubadora a $38{ }^{\circ} \mathrm{C}$ y $5 \%$ de $\mathrm{CO}_{2}$. Se realizaron extracciones de ARN total en cada muestra de cultivo celular en el tiempo asignado empleando el kit RNeasy Mini Kit (Qiagen) de acuerdo con las instrucciones del fabricante y se procedió a guardar el tubo con la muestra a $-80{ }^{\circ} \mathrm{C}$.

Se cuantificó la concentración de ARNm en cada muestra mediante el kit de cuantificación Quant-iT ${ }^{\mathrm{TM}}$ RNA Assay Kit, broad range (ThermoFisher Scientific) y con un equipo de cuantificación Qubit ${ }^{\circledR} 2.0$ Fluorometer. Las muestras fueron cuantificadas y uniformizadas mediante diluciones para llegar a una concentración aproximada 
de $2 \mathrm{ng} / \mu$ le ARN total, lo que hizo un total de 4 ng por reacción para la síntesis de $\mathrm{ADNc}$ usados en las pruebas de qPCR. Luego, mediante el kit Maxima First Strand cDNA Synthesis Kit for RT-qPCR (ThermoFisher Scientific) se sintetizó el ADNc para su uso en el qPCR.

El RT-PCR en tiempo real fue realizado en un termociclador PikoReal 96 (Thermo Scientific), usando como fluoróforo Maxima SYBR Green. Se utilizó el ADNc (4 ng, aproximadamente) sintetizado a partir del ARNm obtenido durante la extracción de los cultivos celulares incubados en diferentes tiempos. Para la prueba de PCR en tiempo real se utilizaron un Maxima SYBR Green/ ROX qPCR Master Mix (2X) a concentración $1 \mathrm{X}$, cebadores específicos para cada citoquina y el normalizador (Cuadro 1) a concentraciones de $0.3 \mu \mathrm{M}, 2 \mu 1$ de ADNc y completando con agua ultra pura libre de nucleasas hasta un volumen de $20 \mu$ l.

En la qPCR se llevó a $50{ }^{\circ} \mathrm{C}$ por 2 min $\left(\mathrm{T}_{\mathrm{DG}}\right)$, luego una temperatura de desnaturalización $\left(\mathrm{T}_{\mathrm{D}}\right.$ ) inicial de $95^{\circ} \mathrm{C}$ por $10 \mathrm{~min}$, luego 40 ciclos de desnaturalización $\left(\mathrm{T}_{\mathrm{D}}\right)$ a $95^{\circ} \mathrm{C}$ por $35 \mathrm{~s}$, hibridación $\left(\mathrm{T}_{\mathrm{A}}\right)$ a la temperatura que corresponde a cada cebador por $30 \mathrm{~s} \mathrm{y}$ una extensión $\left(\mathrm{T}_{\mathrm{E}}\right)$ de $72{ }^{\circ} \mathrm{C}$ por $35 \mathrm{~s}$ (40 en caso de GAPDH). Finalmente, una evaluación de la temperatura de disociación $\left(\mathrm{T}_{\mathrm{MELT}}\right)$, que va desde $\left\langle\mathrm{X} »-95{ }^{\circ} \mathrm{C}\right.$, donde $\langle\mathrm{X}\rangle$ corresponde a la $\mathrm{T}_{\mathrm{A}}$ de cada gen evaluado.

\section{Cuantificación Relativa de los Niveles de Expresión de la Citoquinas}

Para la cuantificación relativa de las citoquinas fue usado el método del Ct comparativo $2^{-\Delta \Delta C t}$ (Livak y Schmittdgen, 2001), que permite comparar las curvas de amplificación de las citoquinas de los tratamientos frente a las curvas de amplificación de las citoquinas producidas en el grupo control.

\section{Resultados}

Todos los ensayos mostraron la presencia de ARNm de GAPDH por PCR en tiempo real, lo cual fue corroborado en gel de agarosa al $2 \%$, observándose un producto único de aproximadamente $356 \mathrm{pb}$ similar al descrito por Patil et al. (2004). Igualmente, la electroforesis en gel de agarosa al $2 \%$ de los productos del PCR en tiempo real para TNF- $\alpha$, IL-10, IL-4, IL-2 e IFN- $\gamma$ mostraron una banda única y específica de ADN amplificado.

Se observa un mayor incremento en la expresión de TNF- $\alpha$ e IL-10, sobre todo a las 48 horas. Una menor expresión de IFN- $\gamma$ e IL-4 entre las 24 y 48 horas y finalmente poca expresión de IL-2 llegando a su pico a las 48 horas, pero cerca a niveles basales (Figura 1).

\section{Discusión}

La P6-like recombinante muestra una tendencia a estimular las citoquinas del perfil Th2. Siendo una proteína de membrana externa es razonable que estimule un perfil inmunológico neutralizante que actúe frente a este tipo de antígeno, pero además estimula la expresión de TNF- $\alpha$. Esto puede ser parte de una respuesta innata donde el TNF- $\alpha$ también forma parte, promoviendo la migración de macrófagos y neutrófilos al sitio de infección característicos de neumonías causadas por P. multocida (Guzmán, 2011; Cirilo et al., 2012; Guzmán et al., 2013; Tizard, 2013), aunque no se descarta que otros factores de virulencia estén implicados como es el caso del lipopolosacárido (LPS) (Harper et al., 2006). Este resultado es muy distinto al presentado por More (2013) que evaluó la expresión de citoquinas frente a una combinación de antígenos clostridiales con ácido retinoico en intestino de alpacas, donde IFN$\gamma$ e IL-2 aumentaban su expresión en la pri- 




Figura 1. Niveles de expresión relativa de las cinco citoquinas evaluados con respecto al tiempo de exposición, evidenciándose una marcada expresión de IL-10 y TNF- $\alpha$

mera semana, posiblemente debido al uso de todos los antígenos clostridiales por parte de dicho estudio.

En el caso del TNF- $\alpha$ posiblemente se deba a que esta citoquina también forma parte del perfil de respuesta innata que promueve la migración de otras células del sistema inmune (Tizard, 2013). Este incremento puede deberse a la presencia de otros tipos de células presentes en los cultivos, como son células inmunes innatas (ejemplo: Natural Killer cells y monocitos) que están siendo estimuladas por la P6-like. Es así, que se observa un aumento de la expresión de TNF- $\alpha$, similar al estudio de Yoo et al. (1995) donde se estimularon macrófagos alveolares bovinos con lipopolisacárido de $P$. (Manhemmia) haemolytica, observándose un incremento de TNF- $\alpha$ a partir de las $4 \mathrm{~h}$ pos-desafío, llegando a una máxima expresión a las $24 \mathrm{~h}$ de la estimulación.

En el caso de IL-2 se observó un aumento de su expresión en relación al calibrador a partir de las 6 horas, llegando a su mayor expresión a las 48 horas (3.63 veces). Este ligero aumento sería suficiente para la activación de linfocitos maduros y su migración hacia el sitio de inflamación. Se considera que IL2 es uno de los factores de crecimiento más importante de linfocitos $\mathrm{T}$, sobre todo cuando son activados por antígenos, expandiendo su número significativamente (Malek et al., 2002).

IFN- $\gamma$ muestra un aumento a las 3 horas (5.54 veces), manteniéndose en bajos niveles a las 6 y 9 horas pos-exposición, alcanzando un pico máximo ( 10.35 veces) a las 48 horas del desafío. El IFN- $\gamma$ tiene un rol importante en la activación de los macrófagos, respuesta inmunitaria innata y respuesta celular adaptativa, el cual asociado con el TNF- $\alpha$ estimula la migración de monocitos, neutrófilos y linfocitos hacia los pulmones con neumonía pasteurelósica (Schroder et al., 2004, Guzmán, 2011; Cirilo et al., 2012; Guzmán et al., 2013), posiblemente estimulados por la P6-Like recombinante. No obstante, no se descartan otros factores de virulencia de Pasteurella como es el lipopoliscárido (Harper et al., 2006). 
El perfil Th2-IL-10 muestra un aumento en su expresión a las 3 horas de la exposición (2.4 veces), aumentando su expresión hasta las 48 h (35.99 veces) de la exposición. IL-10, también conocida como factor de inhibición de la síntesis de citoquinas, tiene propiedades antiinflamatorias que inhibe la síntesis de citoquinas proinflamatorias producidas por linfocitos T y macrófagos (IL-2, IFN$\gamma$, IL-6, IL-8 e IL-12) en monocitos/ macrófagos y neutrófilos, así como la respuesta de los linfocitos Th1 (Opal y De Palo, 2000). Este aumento en su expresión implicaría una baja en la expresión de IL-2 e IFN$\gamma$, dado su carácter inhibidor, aunque este último tiene un aumento en su expresión, por lo que aun expresándose esas dos citoquinas, todavía están bajo influencia de la IL-10 por su carácter inmunoregulador sobre ellas (Neurath et al., 2002).

IL-4 muestra un aumento de su expresión a las 3 horas (4.10 veces) para luego mantenerse constante y aumentar a las 18 horas (3.10 veces), llegando a un pico máximo a las 24 h (9.76 veces). IL-4 estimula la diferenciación de linfocitos a un perfil Th2, de manera autocrina favoreciendo su propia diferenciación. IL-4 inhibe la diferenciación de las células Th1 al regular de manera negativa la producción de IL-12 a partir de macrófagos. Se ha demostrado que es capaz de bloquear o suprimir a las citoquinas derivadas de macrófagos como IL- 1 , TNF- $\alpha$, IL-6 e IL-8 (Berin et al., 1999; Opal y De Palo, 2000). Se observa una mayor expresión de IL-4 a las 24 y 48 horas de la exposición a la proteína P6-like recombinante, pero se deduce que es insuficiente para suprimir la expresión de TNF- $\alpha$ que a las 48 horas tiene su mayor pico de expresión.

En un estudio sobre la diferencia de expresión del perfil Th1 y Th2 en PBMC de búfalos frente al peptidoglicano del Bacillus subtilis se observó una tendencia al perfil Th1 con respecto a Th2, mostrándonos reducción de la expresión de IL-4 e IL-10 y un aumento notorio de TNF- $\alpha$ e IFN- $\gamma$ (Shah et al., 2012). Esta diferencia probablemente se deba a la naturaleza de las proteínas, ya que la P6like es una proteína de membrana externa de la $P$. multocida, mientras que el peptidoglicano es componente de la pared celular del B. subtilis.

En un estudio sobre estimulación citocínica de PBMC bovinas frente a un antígeno de una vacuna inactivada del virus de la fiebre aftosa se evaluó el perfil citocínico de IL-2, IL-4 e IFN- $\gamma$, observándose altos niveles de expresión a las seis horas en IL-2 e IFN- $\gamma$, en tanto que IL-4 llegó a su máxima expresión a las 24 horas de la inducción, encontrando que su perfil citocínico tiene una tendencia al perfil Th1 (Dar et al., 2015). Esto difiere a los hallazgos encontrados en este estudio donde la mayoría de picos de expresión se obtienen a las 48 horas. Además, en dicho estudio se esperaba una tendencia al perfil $\mathrm{Th} 2$, debido a que es una proteína procedente de una vacuna inactivada, pero se observó una tendencia al perfil Th1 por el aumento de la citoquinas de este perfil, y una baja expresión de la IL-4, difiriendo con los resultados del presente estudio donde IL-2 e IFN- $\gamma$ manifiestan una baja expresión.

Boeuf et al. (2005) evaluaron la eficiencia en una técnica de cuantificación absoluta de citoquinas en humanos mediante RTqPCR, donde estimularon PBMC humanas con lipopolisacárido y una Staphylococcus aureus cepa Cowan (SAC), encontrando que la citoquina IL-10 tuvo un aumento en su expresión a partir de las 6 horas llegando a su máximo a las 9 horas ( 10 veces su expresión basal), en comparación con el presente estudio donde IL-10 tuvo un aumento considerable de su expresión a las 12 horas y una mayor expresión a las 48 horas. Asimismo, en dicho estudio, IL-4 muestra un pico de expresión ( 20 veces) a las 2 horas, tanto a estímulo de LPS como de SAC, en tanto que en el presente estudio tuvo una menor expresión relativa; por otro lado, TNF- $\alpha$ mostró un aumento considerable en su expresión a las 3 horas (100 veces con SAC y 50 veces con LPS), mientras que en el presente estudio llegó a su mayor expresión a las 48 horas (30 ve- 
ces). La diferencia entre estas expresiones puede deberse a factores como la naturaleza antigénica del estímulo como al LPS.

En otro estudio sobre la cinética de producción de citoquinas en PBMCs humanas en respuesta al ADN de Brucella se observó que la producción de citoquinas de IL-10 no aumentó hasta el día 4, mientras que IL-2 e IFN- $\gamma$ presentaron un aumento en su concentración con el pasar de los días, observándose una clara tendencia hacia un perfil Th1 (Lashkarbolouki et al,. 2005), diferente a los hallazgos encontrados en el presente estudio, donde IL-2 e IFN- $\gamma$ muestran bajas expresiones e IL-10 aumenta su expresión con el tiempo.

En un estudio reciente, Shivachandra et al. (2017) evaluaron la inmunogenicidad de una Omp16 recombinante de Pasteurella multocida $\mathrm{B}: 2$ en modelo ratón, observando una tendencia a la producción de anticuerpos específicos por parte de los animales inoculados con el antígeno recombinante, similar enunciado que se indica en el presente estudio.

\section{Conclusiones}

- La proteína P6-Like recombinante de Pasteurella multocida aislado de casos fatales de neumonías en crías de alpaca estimula la expresión de citoquinas de respuesta inmune Th1 (TNF- $\alpha$, IFN- $\gamma$ e IL-2) y Th2 (IL-10 e IL-4) en células mononucleares periféricas sanguíneas (PBMC) de alpaca, pero difiriendo en su intensidad.

- La proteína P6-Like recombinante estimula una respuesta Th2 en PBMC de alpacas.

\section{Agradecimientos}

Los autores expresan su agradecimiento al Programa Nacional de Innovación para la Competitividad y Productividad - Innóvate Perú, fuente financiadora del Proyecto
«Vacunología reversa: desarrollo de una vacuna de nueva generación para el control y/o prevención de la neumonía pasteurelósica en alpacas», Contrato $\mathrm{N}^{\circ} 133-\mathrm{FINCyT}$-IB-2013; al CIP-La Raya de la Facultad de Medicina Veterinaria y Zootecnia de la Universidad Nacional del Altiplano; y al Laboratorio de Biotecnología Reproductiva de la FMVUNMSM por su apoyo al trabajo desarrollado.

\section{Literatura Citada}

1. Ameghino E, DeMartíni J. 1991. Mortalidad de crías de alpacas. Boletín de Divulgación del Instituto Veterinario de Investigaciones Tropicales y de Altura (IVITA). Lima, Perú: UNMSM. 128 p.

2. Berin MC, Yang P, Ciok L, Waserman S, Perdue MH. 1999. Role for IL-4 in macromolecular transport across human intestinal epithelium of permeability. Am J Physiol 276: 1046-1052.

3. Boeuf P, Vigan-Womas I, Jublot D, Loizon S, Barale J, Akanmori B, Mercereau-Puijalon O, Behr C. 2005. CyProQuant-PCR: a real time RT-PCR technique for profiling human cytokines, based on external RNA standards, readily automatable for clinical use. BMC Immunology 6: 5. doi: 10.1186/ 1471-2172-6-5

4. Boyce JD, Seemann T, Adler B, Harper M. 2012. Pathogenomics of Pasteurella multocida. Curr Top Microbiol Immunol 361: 23-38. doi: 10.1007/82_2012_203

5. Bustinza $\bar{V} .200 \overline{1}$. La alpaca, conocimiento del gran potencial andino. Puno: Univ. Nacional del Altiplano. 343 p.

6. Cirilo E, Manchego A, Rivera $\mathrm{H}$, Rosadio R. 2012. Coexistencia de virus y bacterias en neumonías agudas en alpacas neonatas. Rev Inv Vet Perú 23: 317-335. doi: 10.15381/rivep.v23i3.914

7. Dar PA, Hajam IA, Suryanarayana VS, Kishore S, Kondabattula G 2015. Kinetics of cytokine expression in bovine PBMMCs and whole blood after in vitro 
stimulation with foot-and-mouth disease virus (FMDV) antigen. Cytokine 72: 5862. doi: 10.1016/j.cyto.2014.12.011

8. Garmendia A, Palmer G, DeMartini J, McGuire T. 1986. Failure of passive immunoglobulin transfer: a major determinant of mortality in newborn alpacas (Lama pacos). Am J Vet Res 48 1472-1476.

9. Guzmán K, Rosadio R, Maturrano L, Manchego A. 2013. Asociación de agentes virales y bacterianos en cuadros de neumonías agudas en alpacas tuis. Rev Inv Vet Perú 24: 524-536.

10. Guzmán KP. 2011. Identificación de polimorfismos del gen TLR4 en crias de alpacas con cuadros de neumonías por Pasteurella multocida. Tesis de Médico Veterinario. Lima, Perú: Univ. Nacional Mayor de San Marcos. 70 p.

11. Harper M, Boyce JD, Adler B. 2006. Pasteurella multocida pathogenesis: 125 years after Pasteur. FEMS Microbiol Lett 265: 1-10. doi: 10.1111/j.15746968.2006.00442.x

12. Harper M, John M, Turni C, Edmunds M, St Michael F, Adler B, Blackall PJ, et al. 2015. Development of a rapid multiplex PCR assay to genotype Pasteurella multocida strains by use of the lipopolysaccharide outer core biosynthesis locus. J Clin Microbiol 53: 477-85. doi: 10.1128/JCM.02824-14

13. Heddleston KL, Gallagher JE, Rebers $P A$. 1972. Fowl cholera: gel diffusion precipitin test for serotyping Pasteruella multocida from avian species. Avian Dis 16: 925-936.

14. Kasten RW, Hansen LM, Hinojoza J, Bieber D, Ruehl WW, Hirsh DC. 1995. Pasteurella multocida produces a protein with homology to the P6 outer membrane protein of Haemophilus influenzae. Infect Immun 63: 989-993.

15. Kedrak A, Borkowska-Opacka B. 2003. Immunological response to outer membrane proteins of Pasteurella multocida serotype A: 3 in calves. Bull Vet Inst Pulawy 47: 387-394.
16. Lashkarbolouki T, Ardestani S, Kariminia A, Ziaee A, Torkabadi E, Ebrahimi M. 2005. Kinetic study of cytokines production by human peripheral blood mononuclear cells in response to Brucella DNA. Microbiol Res 163: 466472. doi: 10.1016/j.micres.2006.07.012

17. Livak KJ, Schmittgen TD. 2001. Analysis of relative gene expression data using real time quantitative PCR and the $2^{-\Delta \Delta \mathrm{Ct}}$ method. Methods 25: 402-408. doi: 10.1006/meth.2001.1262

18. Lu YS, Lai WC, Pakes SP, Nie LC. 1991. A monoclonal antibody against a Pasteurella multocida outer membrane protein protects rabbits and mice against pasteurellosis. Infect Immun 59: 172- 180.

19. Malek TR, Yu A, Vincek V, Scibelli P, Kong L. 2002. CD4 regulatory T cells prevent lethal autoimmunity in IL-2Râ deficient mice: implications for the nonredundant function of IL-2. Immunity 17: 167-178. doi: 10.1016/S10747613(02)00367-9

20. More JA. 2013. Efecto de antígenos clostridiales con ácido retinoico sobre la expresión de citoquinas de la respuesta inmune humoral y celular de la mucosa intestinal de crías de alpacas (Vicugna pacos). Tesis de Magister. Lima, Perú: Univ. Nacional Mayor de San Marcos. $104 \mathrm{p}$.

21. Neurath M, Weigmann B, Finotto $S$, Glickman J, Nieuwenhuis R, Iijima H, Mizoguchi A, et al. 2002. The transcription factor T-bet regulates mucosal $\mathrm{T}$ cell activation in experimental colitis and Crohn's disease. J Exp Med 195: 1129-1143. doi: 10.1084/ jem.20011956

22. Odbileg R, Konnai S, Ohashi K, Onuma M. 2005. Molecular cloning and phylogenetic analysis of inflammatory cytokines of Camelidae (llama and camel). J Vet Med Sci 67: 921-925.

23. Odbileg R, Purevtseren B, Batsukh Z, Konnai S, Ohashi K, Onuma M. 2006. Complete cDNA sequences and phylogenetic analysis of the Th1 and Th2 
cytokines of the bactrian camel (Camelus bactrianus). J Vet Med Sci 68: 941-946. doi: 10.1292/jvms.68.941

24. Odbileg R, Purevtseren B, Gantsetseg D, Boldbaatar B, Buyannemekh T, Galmandakh Z, Erdenebaatar J, et al. 2008. Cytokine responses in camels (Camelus bactrianus) vaccinated with Brucella abortus strain 19 vaccine. J Vet Med Sci 70: 197-201.

25. Opal SM, De Palo VA. 2000. Antiinflammatory cytokines. Chest 117: 11621172. doi: $10.1378 /$ chest.117.4.1162

26. Patil A, Hughes A, Zhang G. 2004. Rapid evolution and diversification of mammalian $\alpha$-defensins as revealed by comparative analysis of rodent and primate genes. Physiol Genomics 20: 1-11. doi: 10.1152/physiolgenomics. 00150.2004

27. Rosadio R, Ameghino E, Ramírez A. 1990. Diagnosis and control of diseases in sheep and alpaca in Peru. In: Improving Andean sheep and alpaca production. Mc Corkle CM (ed). USA: University of Missoury-Columbia Printing Services. p 141-220.

28. Schroder K, Hertzog P, Ravasi T, Hume D. 2004. Interferon: an overview of signals, mechanisms and functions. J Leukoc Biol 75: 163-189. doi: 10.1189/ jlb.0603252
29. Shah SM, Kumar R, Brah GS, Santra L, Pawar H. 2012. Differential expression of Th1- and Th2- type cytokines in peripheral blood mononuclear cells of Murrah buffalo (Bubalus bubalis) on TLR2 induction by $B$. subtilis peptidoglycan. Asian-Australas J Anim Sci 25: 1021-1028. doi: 10.5713/ ajas.2012.12033

30. Shivachandra S, Kumar A, Mohanty $N$, Yogisharadhya R. 2017. Immunogenicity of recombinant Omp 16 protein of Pasteurella multocida B:2 in mouse model. Indian J Anim Sci 87: 29-34.

31. Tizard IR. 2013. Veterinary immunology. $9^{\text {th }}$ ed. Philadelphia, USA: Elsevier. $568 \mathrm{p}$.

32. Yoo H, Maheswaran SK, Lin G, Townsend EL, Ames TR. 1995. Induction of inflammatory cytokines in bovine alveolar macrophages following stimulation with Pasteurella haemolytica lipopolysaccharide. Infect Immun 63:381-388.

33. Zhang H, Ainsworth AJ, Montgomery RD. 1994. Use of a $35.5 \mathrm{kDa}$ cell membrane composition of Pasteurella multocida and an anti-idiotype antibody to induce protective immunity in Leghorn chickens. Vet Immunol Immunopathol 41:89-100. 